\title{
Dynamic Semiparametric Factor Models in Risk Neutral Density Estimation
}

\author{
Enzo Giacomini* \\ Wolfgang Härdle* \\ Volker Krätschmer**
}

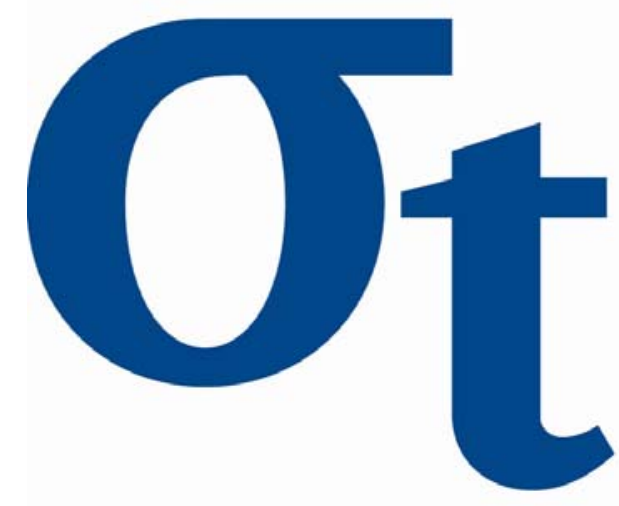

)

$\nabla$

6

* Humboldt-Universität zu Berlin, Germany

** Technische Universität Berlin, Germany 


\title{
Dynamic Semiparametric Factor Models in Risk Neutral Density Estimation
}

\author{
Enzo Giacomini ${ }^{\text {a,* }}$, Wolfgang Karl Härdle ${ }^{a}$, \\ Volker Krätschmer ${ }^{\mathrm{a}, \mathrm{b}}$ \\ ${ }^{a}$ Humboldt-Universität zu Berlin, \\ CASE - Center for Applied Statistics and Economics \\ Spandauerstr. 1, 10178 Berlin, Germany \\ ${ }^{\mathrm{b}}$ Technische Universität Berlin, Institute of Mathematics \\ Straße des 17. Juni 136, 10623 Berlin, Germany
}

\begin{abstract}
Dimension reduction techniques for functional data analysis model and approximate smooth random functions by lower dimensional objects. In many applications the focus of interest lies not only in dimension reduction but also in the dynamic behaviour of the lower dimensional objects. The most prominent dimension reduction technique - functional principal components analysis - however, does not model time dependences embedded in functional data. In this paper we use dynamic semiparametric factor models (DSFM) to reduce dimensionality and analyse the dynamic structure of unknown random functions by means of inference based on their lower dimensional representation. We apply DSFM to estimate the dynamic structure of risk neutral densities implied by prices of option on the DAX stock index.
\end{abstract}

Key words: dynamic factor models, dimension reduction, risk neutral density AMS classification: $62 \mathrm{G} 08,62 \mathrm{M} 10,62 \mathrm{P} 05$

JEL classification: C14, C32, G12

\section{Introduction}

Large datasets containing various samples of high dimensional observations became common in diverse fields of science with advances in measurement and computational techniques. In many applications the data come in curves, i.e.

\footnotetext{
* Corresponding author. Tel. + 49302093 5721, Fax. + 493020935649

Email address: giacomini@wiwi.hu-berlin.de (Enzo Giacomini).
} 
as observations of discretized values of smooth random functions, presenting evident functional structure. In these cases it is natural to perform statistical inference using functional data analysis techniques.

Consider a dataset $\left\{\left(Y_{j t}, X_{j t}\right)\right\}, j=1, \ldots, J_{t}, t=1, \ldots, T$ containing noisy samples of a real valued smooth random function $\mathcal{F} \in L_{2}(\mathcal{X}), \mathcal{X} \subseteq \mathbb{R}^{d}, d \in \mathbb{N}$ evaluated at unbalanced design points $X_{j t}$ as

$$
Y_{j t}=\mathcal{F}_{t}\left(X_{j t}\right)+\varepsilon_{j t}
$$

where $\varepsilon_{j t}$ denotes unknown zero-mean error terms and $\left\{\mathcal{F}_{t}\right\}$ are i.i.d. realizations of $\mathcal{F}$. Each sample $S_{t}=\left\{\left(Y_{j t}, X_{j t}\right): j=1, \ldots, J_{t}\right\}, t=1, \ldots, T$ may correspond to a different observation on e.g. a different individual, time period or experimental condition.

Examples in biomedicine are measurements across individuals of growth curves or of brain potentials obtained from EEG, see Kneip and Gasser (1992) and Gasser and Kneip (1995). In econometrics data may originate from expenditures on commodities across households, Kneip (1994), implied volatilities observed on different trading days, Fengler, Härdle, and Mammen (2007) or measurements on stock liquidity across time, Dähne, Härdle, and Hautsch (2008). Ramsay and Silverman (2005) and Ferraty and Vieu (2006) provide an extensive list of functional datasets encountered in various applications.

A large branch of functional data analysis concentrates on modelling and approximating the random function $\mathcal{F}$ by lower dimensional objects. Distributions on function spaces are highly complex objects and dimension reduction techniques present a feasible and interpretable approach to investigate them. Functional principal components analysis (FPCA), based on the KarhunenLoève expansion of $\mathcal{F}$, is the most prominent and widely used dimension reduction technique, see Rao (1958), Rice and Silverman (1991) and Ramsay and Dalzell (1991).

Asymptotic results on FPCA have been obtained by Dauxois, Pousse, and Romain $(1982)$ for directly observed functional data $\left\{\mathcal{F}_{t}\left(X_{j t}\right)\right\}$. In cases where functional data is not directly observable a preliminary smoothing is required at each $S_{t}$ and FPCA is performed on the smoothed $\left\{\widehat{\mathcal{F}}_{t}\right\}$, see Besse, Cardot, and Ferraty (1997) and Benko, Kneip, and Härdle (2008) for recent developments. In practical applications the previous fits may suffer, however, from design-sparseness at each $S_{t}$, Cont and da Fonseca (2002) and Fengler et al. (2007).

In general lines, previous literature combines PCA and dimension reduction with presmoothing for effective dimensional space at fixed time horizon. Various applications, however, involve modelling the dynamics of the unobserved 
random functions and call for dimension reduction techniques that smooth in space and are parametric in time.

In this paper we simultaneously reduce dimensionality avoiding preliminary smoothing and consider the time structure in the sequence $\left\{\mathcal{F}_{t}\right\}$. We describe $\mathcal{F}_{t}$ as a linear combination of $L+1<<T$ unknown smooth functions, called basis functions, $m_{l} \in L_{2}(\mathcal{X}), l=0, \ldots, L$ :

$$
\mathcal{F}_{t}\left(X_{j t}\right)=\sum_{l=0}^{L} Z_{l t} m_{l}\left(X_{j t}\right)
$$

Here $Z_{t}=\left(Z_{0 t}, \ldots, Z_{L t}\right)^{\top}$ is an unobservable random vector taking values on $\mathbb{R}^{L+1}$. Defining the tuple of functions $m=\left(m_{0}, \ldots, m_{L}\right)^{\top}$, the dynamic semiparametric factor model (DSFM), Borak, Härdle, Mammen, and Park (2008), reads as

$$
Y_{j t}=Z_{t}^{\top} m\left(X_{j t}\right)+\varepsilon_{j t}
$$

The basis functions are estimated nonparametrically avoiding specification issues, i.e. the shape of $m_{l}$ is extracted from the data. As $m$ and $Z_{t}$ are estimated simultaneously, the smoothing is transfered directly to $m_{l}, l=0, \ldots, L$ and design-sparseness becomes secondary. In addition, and essential for investigating dynamics, the random process $\left\{Z_{t}\right\}$ may be non-stationary.

The form 1.2 is justified when prior knowledge about the available dataset leads to expect some common structure generating each $\mathcal{F}_{t}$. Approximating it by a lower dimensional linear combination of common factors is a natural way to handle and describe the unobservable generating mechanism.

In many applications the index $t$ reflects time evolution and the unobservable $\left\{Z_{t}\right\}$ contains the dynamics of $\left\{\mathcal{F}_{t}\right\}$. Borak et al. (2008) show that under (1.2) the autocorrelation structures of estimated $\left\{Z_{t}\right\}$ and true $\left\{Z_{t}\right\}$ are asymptotically equivalent. This result implies that no loss is incurred by inferring the dynamic structure from $\left\{\widehat{Z}_{t}\right\}$, i.e. there is no payment for not knowing the true $\left\{Z_{t}\right\}$. This fact is essential for investigating cointegration with another dynamical systems. An application using financial data is provided by Brüggemann, Härdle, Mungo, and Trenkler (2008) where the cointegration between estimates $\left\{Z_{t}\right\}$ and macroeconomic time series is analysed.

Note that in the very similar common regressors model from Kneip (1994) the unobservable functions are also considered a linear combination of unspecified common functions as in 1.2 and are estimated from the data. There are however crucial differences between DSFM and common regressors model: 

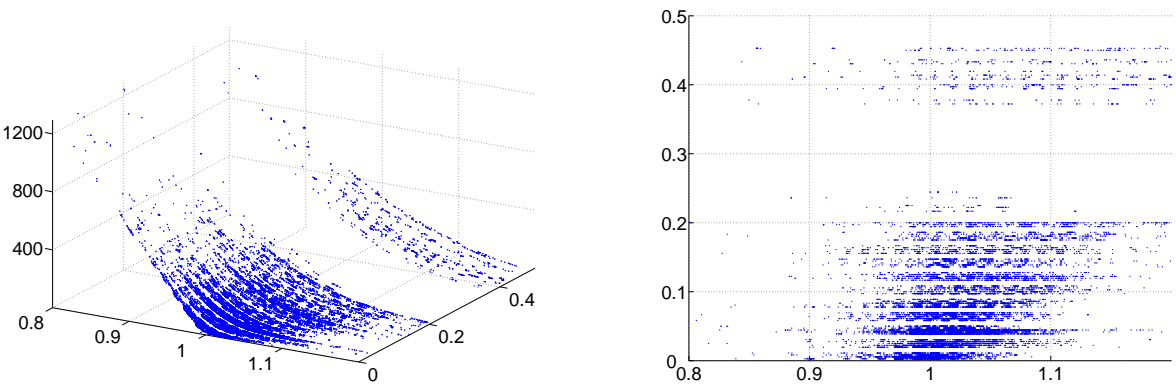

Fig. 1. Samples $S_{t}, t=1, \ldots, 22$ of DAX call prices traded on January 2001 (left). Corresponding unbalanced design $\left\{X_{j t}\right\}$ (right)

(1) in DSFM $\left\{Z_{t}\right\}$ is a (non-stationary) random process with autocovariance structure inferable from $\left\{\widehat{Z}_{t}\right\}$

(2) DSFM is implementable in unbalanced designs

(3) DSFM avoids presmoothing by transferring the smoothing to the basis functions

Hence, in contrast to the dimension reductions techniques FPCA and common regressors, DSFM reduces dimensionality capturing the structural dynamics embedded in the observations.

We apply DSFM on a financial dataset of option prices in order to investigate the dynamics of risk assessments from investors acting in a market. Option prices are a valuable source of information concerning risk assessments from investors about future financial payoffs. The information is summarized in the risk neutral densities q, the continuous counterpart from Arrow-Debreu security prices, see Ait-Sahalia and Lo (1998). Under no arbitrage assumptions the risk neutral densities - corresponding to a risk neutral measure $\mathbb{Q}$ - are derived from prices of European call options.

An European call option on $S_{t}$ with maturity date $T>0$ and strike $K>0$ is a financial instrument that delivers at time $T$ the random payoff $\left(S_{T}-K\right)^{+}$ where $S_{t}$ is the price of the underlying asset at time $0 \leq t \leq T$. Breeden and Litzenberger (1978) show that under no arbitrage assumptions the risk neutral density is obtained from the European call price function $C_{t}$ through the relation

$$
q_{t, T}\left(s_{T} \mid s_{t}\right)=\left.e^{r(T-t)} \frac{\partial^{2} C_{t}\left(s_{t}, K, T-t\right)}{\partial K^{2}}\right|_{K=s_{T}}
$$

where $r>0$ is interest rate, see Section (3) for details.

The knowledge about the risk neutral densities delivers (time dependent) risk assessments from investors concerning future payoffs and is essential for appli- 
cations in financial economics, option pricing and risk management as, e.g. estimated risk neutral densities allow less liquid instruments or contracts traded over-the-counter to be priced. From the economic point of view it is natural to investigate the dynamics of the risk neutral densities and the dependence between risk assessments and macroeconomic and financial indicators. This calls for a method that reduces dimensionality and allows for dynamic analysis of the lower dimensionals. Both features are present in DSFM.

We estimate the risk neutral densities based on a dataset containing intraday prices of calls on the German stock index (DAX) from 2001. Each observation consists of a call price $Y_{j t}$ on a design point $X_{j t}=\left(\kappa_{j t}, \tau_{j t}\right)^{\top}$ where $j=$ $1, \ldots, J_{t}$ denotes the transactions at day $t=1, \ldots, T$ and $\kappa=g(K)$ is the moneyness, a monotone transformation of strikes $K$. Exchange regulations impose prespecified values for tradable maturities $\tau=T-t$ and are responsible for the observed degenerated design, see figure 1 .

Following Ait-Sahalia and Lo (1998) and Fengler et al. (2007) the observations are transformed into log-implied volatilities $Y_{j t}=\log C_{B S}^{-1}\left(Y_{j t}\right)$, see Section (3) for details. These are assumed as discretized noisy values of the log-implied volatility surface evaluated at design points $\left\{X_{j t}\right\}$ :

$$
\tilde{Y}_{j t}=\log \mathcal{V}_{t}\left(X_{j t}\right)+\varepsilon_{j t}
$$

where the smooth random function $\mathcal{V} \in L_{2}(\mathcal{X}), \mathcal{X} \subset \mathbb{R}_{+}^{2}$, is called the implied volatility surface and $\varepsilon_{j t}$ is an error term. The realizations $\left\{\mathcal{V}_{t}\right\}$ are filtered out from the transformed data with DSFM and the risk neutral densities estimated using (1.4) with $C_{B S}\left(\widehat{\mathcal{V}}_{t}\right)$ as an estimator for $C_{t}$. The dynamics of the estimated $\left\{\widehat{q}_{t, T}\right\}$ is analysed based on the autocorrelation structure of $\left\{\widehat{Z}_{t}\right\}$.

In the sequel the DSFM estimation method and its asymptotic properties are described (Section 2). In the application part (Section 3), risk neutral densities are defined and estimated from observed prices of European call options on the DAX index (ODAX dataset). Their dynamic structure is analysed by vector autoregressive models.

\section{Estimation Method}

Consider a dataset $\left\{\left(Y_{j t}, X_{j t}\right)\right\}, j=1, \ldots, J_{t}, t=1, \ldots, T$ such that

$$
Y_{j t}=\sum_{l=0}^{L} Z_{l t} m_{l}\left(X_{j t}\right)+\varepsilon_{j t}
$$


where $\varepsilon_{j t}$ is an unknown error terms with $E\left[\varepsilon_{j t}\right]=0$ and $E\left[\varepsilon_{j t}^{2}\right]<\infty$ and $\left\{\varepsilon_{j t}\right\}$ are independent. Here $Z_{t}=\left(Z_{0 t}, \ldots, Z_{L t}\right)^{\top}$ is an unobservable random vector taking values on $\mathbb{R}^{L+1}$ with $Z_{0 t}=1$ and $m_{l} \in L_{2}(\mathcal{X}), l=0, \ldots, L$ are unknown smooth functions, called basis functions, mapping $\mathcal{X} \subseteq \mathbb{R}^{d}, d \in \mathbb{N}$ into real values.

Following Borak et al. (2008), the basis functions are estimated using a series expansion. Defining $K$ normed functions $\psi_{k}: \mathcal{X} \rightarrow \mathbb{R}, \int_{\mathcal{X}} \psi_{k}^{2}(x) d x=1, k=$ $1, \ldots, K$ and a $(L+1 \times K)$ matrix of coefficients $\Gamma=\left(\gamma_{l, k}\right), \gamma_{l, k} \in \mathbb{R}$, the tuple of functions $m=\left(m_{0}, \ldots, m_{L}\right)^{\top}$ is approximated by $\Gamma^{\top} \psi$ where $\psi=$ $\left(\psi_{1}, \ldots, \psi_{K}\right)^{\top}$. For simplicity of notation we assume that $J_{t}=J$ does not depend on $t$. We define the least square estimators as

$$
(\widehat{\Gamma}, \widehat{Z})=\arg \min _{\Gamma \in \mathcal{G}, Z \in \mathcal{Z}} \sum_{t=1}^{T} \sum_{j=1}^{J}\left\{Y_{j t}-Z_{t}^{\top} \Gamma \psi\left(X_{j t}\right)\right\}^{2}
$$

where $\mathcal{G}=\mathcal{M}(L+1, K), \mathcal{Z}=\left\{Z \in \mathcal{M}(T, L+1): Z_{0 t}=1\right\}$ and $\mathcal{M}(a, b)$ is the set of all $(a \times b)$ matrices. The basis functions $m$ are estimated by $\widehat{m}=\widehat{\Gamma} \psi$.

Theorem (2.1) gives the asymptotic behaviour of the least squares estimators $(\widehat{\Gamma}, \widehat{Z})$.

Theorem 2.1 Suppose that DSFM holds and that $(\widehat{\Gamma}, \widehat{Z})$ is defined by (2.2). Under assumptions (A1)-(A8), see Appendix A, it holds for $K, J \rightarrow \infty$ :

$$
\frac{1}{T} \sum_{1 \leq t \leq T}\left\|\widehat{Z}_{t}^{\top} \widehat{\Gamma}-Z_{t}^{\top} \Gamma^{*}\right\|^{2}=\mathcal{O}_{P}\left(\rho^{2}+\delta_{K}^{2}\right)
$$

See Borak et al. (2008) for the proof. Note that the model (2.1) is only identifiable up to linear transformations. Consider a $(L+1 \times L+1)$ regular matrix $B=\left(b_{m n}\right)$ with $b_{1 n}=\delta_{1 n}$ and $b_{m 1}=\delta_{m 1}$ for $m, n=1, \ldots, L+1$ where $\delta_{m n}=\mathbf{1}(m=n)$. Define $Z_{t}^{*}=B^{\top} Z_{t}, m^{*}=B^{-1} m$, then from 1.2

$$
\begin{aligned}
\mathcal{F}_{t}(X) & =Z_{t}^{\top} m(X) \\
& =Z_{t}^{\top} B B^{-1} m(X) \\
& =Z_{t}^{* \top} m^{*}(X)
\end{aligned}
$$

for $X \in \mathcal{X}$. On the other hand it is always possible to chose orthonormal basis functions by setting $m^{*}=H m$ where $H$ is an orthogonal matrix.

Theorem $(2.2)$ states that for any $\widehat{Z}_{t}$ there exists a random matrix $B$ such that the autocovariances of $\left\{\widehat{Z}_{t}\right\}, \widetilde{Z}_{t}=B^{\top} \widehat{Z}_{t}$ are asymptotically equivalent to the 
autocovariances of the true unobservable $\left\{Z_{t}\right\}$. This equivalence is transfered to classical estimation and testing procedures in the context of e.g. vector autoregressive models and in particular justifies inference based on $\left\{\widehat{Z}_{t}\right\}$ when $\left\{Z_{t}\right\}$ is a VAR process. Define for $H_{t} \in \mathcal{Z}, t=1, \ldots, T: \bar{H}=T^{-1} \sum_{t=1}^{T} H_{t}$, $H_{c, t}=H_{t}-\bar{H}$ and $H_{n, t}=\left(T^{-1} \sum_{s=1}^{T} H_{c, s} H_{c, s}^{\top}\right)^{-1 / 2} H_{c, t}$.

Theorem 2.2 Suppose that DSFM holds and that $(\widehat{\Gamma}, \widehat{Z})$ is defined by 2.2). Under assumptions (A1)-(A11), see Appendix $A$, there exists a random matrix $B$ such that for $h \neq 0, h_{d}=\max (1,1-h), h_{u}=\max (T, T-h)$ and $T \rightarrow \infty$ :

$$
\frac{1}{T} \sum_{t=h_{d}}^{h_{u}} \widetilde{Z}_{c, t}\left(\widetilde{Z}_{c, t+h}-\widetilde{Z}_{c, t}\right)^{\top}-\frac{1}{T} \sum_{t=h_{d}}^{h_{u}} Z_{c, t}\left(Z_{c, t+h}-Z_{c, t}\right)^{\top}=\mathcal{O}_{P}\left(T^{-1 / 2}\right)
$$

where $\widetilde{Z}_{t}=B^{\top} \widehat{Z}_{t}$. Moreover

$$
\frac{1}{T} \sum_{t=h_{d}}^{h_{u}} \widetilde{Z}_{n, t} \widetilde{Z}_{n, t+h}^{\top}-\frac{1}{T} \sum_{t=h_{d}}^{h_{u}} Z_{n, t} Z_{n, t+h}^{\top}=\mathcal{O}_{P}\left(T^{-1 / 2}\right)
$$

See Borak et al. (2008) for the proof. Note that, in contrast to FPCA, DSFM does not require stationarity, neither for $\left\{Z_{t}\right\}$ nor $\left\{\varepsilon_{t}\right\}$, but only weak assumptions on the average behaviour of $Z_{t}$, like being a martingale difference, see Appendix A.

\section{Application}

Consider a financial market with one risky asset and one riskless bond with constant interest rate $r>0$. Let the price of the asset traded on the market be described by the real valued random process $\left\{S_{t}\right\}, t=[0, T], T<\infty$ on a filtered probability space $\left(\Omega,\left\{\mathcal{F}_{t}\right\}, \mathbb{P}\right)$ with $\mathcal{F}_{t}=\sigma\left(S_{u}, u \leq t\right)$ and $\mathcal{F}_{0}=\{\emptyset, \Omega\}$. Assume further no arbitrage in the financial market in the sense that there exists a (risk neutral) probability measure $\mathbb{Q}$ equivalent to $\mathbb{P}$ under which the discounted price process $\left\{e^{-r t} S_{t}\right\}$ is a martingale.

A European call option at strike $K>0$ is a financial instrument that pays $\Psi\left(S_{T}\right)=\left(S_{T}-K\right)^{+}$at time $T$. By the risk-neutral valuation principle w.r.t. $\mathbb{Q}$ the price $C_{t}$ of a European call option at time $t$ is defined to be

$$
C_{t}=e^{-r(T-t)} E^{\mathbb{Q}}\left[\Psi\left(S_{T}\right) \mid \mathcal{F}_{t}\right]
$$


Assuming that $\left\{S_{t}\right\}$ is a $\mathbb{Q}$-Markov process and denoting the $\mathbb{P}$-density of $\mathbb{Q}$ by $\pi$, the price can be rewritten as

$$
\begin{aligned}
C_{t}\left(S_{t}\right) & =e^{-r(T-t)} E^{\mathbb{Q}}\left[\Psi\left(S_{T}\right) \mid S_{t}\right] \\
& =e^{-r(T-t)} E\left[\Psi\left(S_{T}\right) \mathcal{K}_{\pi}^{t}\left(S_{t}, S_{T}\right) \mid S_{t}\right]
\end{aligned}
$$

where $E$ denotes the expectation under $\mathbb{P}$ and $\mathcal{K}_{\pi}^{t}\left(S_{t}, S_{T}\right) \stackrel{\text { def. }}{=} \frac{E\left[\pi \mid S_{t}, S_{T}\right]}{E\left[\pi \mid S_{t}\right]}$. The conditional risk neutral distribution of $S_{T}$ is defined as

$$
Q_{S_{T} \mid S_{t}=s_{t}}\left(\left[S_{T} \leq x\right]\right) \stackrel{\text { def. }}{=} \int_{-\infty}^{x} \mathcal{K}_{\pi}^{t}\left(s_{t}, \cdot\right) d P_{S_{T} \mid S_{t}=s_{t}}
$$

where $P_{S_{T} \mid S_{t}=s_{t}}$ is the conditional distribution of $S_{T}$ under $S_{t}=s_{t}$. Specializing to the following two factor model we assume that the price process has dynamics given by

$$
d S_{t}=S_{t} \mu\left(Y_{t}\right) d t+S_{t} \sigma\left(Y_{t}\right) d W_{t}^{1}
$$

here $W^{1}$ is a standard $\mathbb{P}$-Brownian motion and $Y$ denotes an external economic factor process modelled by

$$
d Y_{t}=g\left(Y_{t}\right)+\rho d W_{t}^{1}+\bar{\rho} d W_{t}^{2}
$$

where $\rho \in[-1,1]$ is some correlation factor, $\bar{\rho} \stackrel{\text { def. }}{=} \sqrt{1-\rho^{2}}$ and $W^{2}$ is a standard $\mathbb{P}$-Brownian motion independent of $W^{1}$ under $\mathbb{P}$. Market models of this type are popular in mathematical finance and economics, in particular if $Y$ follows and Ornstein-Uhlenbeck dynamic with mean reversion term $g(y)=$ $\kappa(\theta-y)$ for constants $\theta \geq 0$ and $\kappa>0$. Moreover, $\left\{S_{t}\right\}$ is a $\mathbb{Q}$-Markov process for any $\mathbb{Q}$, see Hernández-Hernández and Schied (2007) and the conditional risk neutral distribution $Q_{S_{T} \mid S_{t}=s_{t}}$ has a density function denoted by $q_{t, T}\left(\cdot \mid s_{t}\right)$. Hence, the call prices $C$ can be expressed as

$$
C_{t}\left(s_{t}, K, T-t\right)=e^{r(T-t)} \int\left(s_{T}-K\right)^{+} q_{t, T}\left(s_{T} \mid s_{t}\right) d s_{T} .
$$

We assume that the observed prices in the financial market are built based on the risk neutral valuation principle w.r.t. an unknown risk neutral measure $\mathbb{Q}$. Our interest lies in estimating the conditional risk neutral distribution $Q_{S_{T} \mid S_{t}=s_{t}}$, or equivalently its density function $q_{t, T}\left(\cdot \mid s_{t}\right)$, implied by $\mathbb{Q}$ through (3.1). 


\subsection{Estimation}

Adapting Breeden and Litzenberger (1978), one can show that the density function $q_{t, T}\left(\cdot \mid s_{t}\right)$, called from now on the risk neutral density, is obtained as the second derivative of the call price function $C_{t}$ with respect to strike $K$

$$
q_{t, T}\left(s_{T} \mid s_{t}\right)=\left.e^{r \tau} \frac{\partial^{2} C_{t}\left(s_{t}, K, \tau\right)}{\partial K^{2}}\right|_{K=s_{T}}
$$

here $\tau=T-t$ is the time to maturity. The price function can be smoothed out of observations from call prices and used in (3.2) to recover state price densities implied on the data.

Following Ait-Sahalia and Lo (1998) smoothing is carried out in the space of implied volatilities. In general the exact form of $C_{t}$ is hard to determine and based on historical data an estimate of $C_{t}$ can be obtained by non-parametric regression. In order to cope with the course of dimensionality, Ait-Sahalia and Lo (1998) proposed a semiparametric approach using the Black-Scholes formula evaluated with a non-parametric estimator for the implied volatility in place of the true non-observable volatility.

The implied volatility surface is the function $\sigma_{t}: \mathbb{R}_{+}^{2} \rightarrow \mathbb{R}_{+}$satisfying for all $(K, \tau) \in \mathbb{R}_{+}^{2}$

$$
C_{t}\left(s_{t}, K, \tau\right)=C_{B S}\left\{s_{t}, r_{t}, K, \tau, \sigma_{t}(K, \tau)\right\}
$$

where $C_{B S}(s, r, K, \tau, \sigma)=s \Phi\left(d_{1}\right)-K e^{-r \tau} \Phi\left(d_{2}\right)$ is the Black-Scholes price of $\Psi$ with strike $K$ and maturity $\tau$. Here $\Phi(x)$ is the standard normal cdf, $d_{1}(\sigma)=\left\{\log \left(\frac{s}{K}\right)+\left(r+\frac{1}{2} \sigma^{2}\right) \tau\right\} /(\sigma \sqrt{\tau})$ and $d_{2}(\sigma)=d_{1}-\sigma \sqrt{\tau}$. Note that $C_{B S}(v)=C_{B S}(s, r, K, \tau, v)$ is a continuous increasing function of $v$, hence $\sigma_{t}(K, \tau)=C_{B S}^{-1}\left\{C_{t}\left(s_{t}, K, \tau\right)\right\}$.
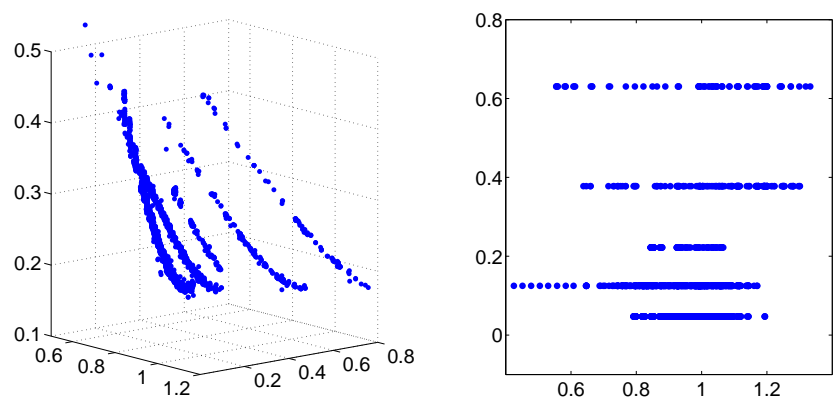

Fig. 2. Call and put implied volatilities observed (left), data design (right), ODAX on 20000502 
More generally, the implied volatility surface is considered a smooth random function $\mathcal{V} \in L_{2}(\mathcal{X})$ on the space $\mathcal{X} \subset \mathbb{R}^{2}$ of strikes $K$ and maturities $\tau$. Combining (3.2) and (3.3) the functional random variable $\mathcal{H} \in L_{2}(\mathcal{X})$ called the risk neutral (RN) surface is defined as

$$
\mathcal{H}=e^{r \tau} D^{2} C_{B S}(\mathcal{V})
$$

where $D^{m}$ denotes the $m$-th derivative with respect to $K$. Clearly lower dimension objects describing $\mathcal{V}$ may be used to analyse $\mathcal{H}$.

A functional dataset containing realizations of $\mathcal{V}$ is however not available, as in an exchange only discretized values of $\mathcal{V}_{t}$ corrupted by noise are registered from trades. More specifically, on each day $t=1, \ldots, T$ there are $J_{t}$ options traded, each intra-day trade $j=1, \ldots, J_{t}$ corresponds to an observed option price $Y_{j t}$ at a pair of moneyness and maturities $X_{j t}=\left(\kappa_{j t}, \tau_{j t}\right)^{\top}$ where $\kappa=e^{r \tau} K / S$. The observed implied volatilities at the respective design points are obtained by $\sigma_{j t}=C_{B S}^{-1}\left(Y_{j t}\right)$, see figure (2). For numerical tractability we perform a further transformation into $\log$-implied volatilities $\tilde{Y}_{j t}=\log \sigma_{j t}$ and use DSFM to model

$$
\tilde{Y}_{j t}=\log \mathcal{V}_{t}\left(X_{j t}\right)+\varepsilon_{j t}
$$

where $\log \mathcal{V}_{t}=Z_{t}^{\top} m$. The implied volatility surface at $t$ is estimated by $\widehat{\mathcal{V}}_{t}=$ $\exp \left(\widehat{Z}_{t}^{\top} \widehat{\Gamma} \psi\right)$, recall $(2.2)$. The RN surface is estimated using $(3.4)$ by $\widehat{\mathcal{H}}_{t}=$ $\mathcal{H}\left(\widehat{\mathcal{V}}_{t}\right)$ where

$$
\mathcal{H}(\mathcal{V})=\varphi\left(d_{2}\right)\left\{\frac{1}{K \sqrt{\tau} \mathcal{V}}+2 d_{1} \frac{D \mathcal{V}}{\mathcal{V}}+K \sqrt{\tau} d_{1} d_{2} \frac{(D \mathcal{V})^{2}}{\mathcal{V}}+K \sqrt{\tau} D^{2} \mathcal{V}\right\}
$$

and $d_{1}=d_{1}(\mathcal{V})$ and $d_{2}=d_{2}(\mathcal{V})$. The dynamics of the unobservable sequence of RN surfaces $\left\{\mathcal{H}_{t}\right\}$ implied in the observations $\left\{\left(Y_{j t}, X_{j t}\right)\right\}$ may be investigated by analysing the lower dimensional $\left\{\widehat{Z}_{t}\right\}$.

\subsection{Results}

Here implied volatility and RN surfaces are estimated with DSFM from intraday call prices on the DAX index, i.e. $S_{t}$ represents the value of the DAX index at time $t$. The time ranges from 20010101 to 20020101 (dates are written as year, month, day) corresponding to 253 trading days.

Tensor B-splines, quadratic in $\tau$ and cubic in $\kappa$ directions placed on $8 \times 6$ knots, are used for the series estimators of $m$. The dimension $L$ of $Z_{t}$ is chosen based on 


$$
R V(L)=\frac{\sum_{t=1}^{T} \sum_{j=1}^{J_{t}}\left\{\tilde{Y}_{j t}-\widehat{Z}_{t}^{\top} \widehat{m}\left(X_{j t}\right)\right\}^{2}}{\sum_{t=1}^{T} \sum_{j=1}^{J_{t}}\left(\tilde{Y}_{j t}-\bar{Y}\right)^{2}}
$$

where $\bar{Y}=\left(\sum_{t=1}^{T} \sum_{j=1}^{J_{t}} \tilde{Y}_{j t}\right) / \sum_{t=1}^{T} J_{t}$. The value $1-R V(L)$ may be interpreted as the ratio of variation explained by the model to total variation. The order of the splines and number of knots have negligible influence on $R V(L)$, as established by inumerous simulations in Borak et al. (2008). The implied volatility and RN surfaces are estimated with DSFM as in (3.5) with $L=3$. Table 1 shows that the addition of the fourth or fifth basis function results in negligible model fit improvement.

\begin{tabular}{cccccc}
\hline \hline$L$ & 1 & 2 & 3 & 4 & 5 \\
\hline $1-R V(L)$ & 0.77 & 0.97 & 0.98 & 0.98 & 0.98 \\
\hline \hline
\end{tabular}

Table 1

Number of basis functions and explained variation

Figures 3 and 4 depict the estimated loading factors series $\left\{\widehat{Z}_{t}\right\}$ and basis functions $\widehat{m}_{l}$. From 3.5 we obtain a sequence of RN surfaces $\left\{\widehat{\mathcal{H}}_{t}\right\}, t=$ $1, \ldots, 253$, figure 5 shows $\widehat{\mathcal{H}}_{t}$ at $t$ corresponding to day 20010710 .

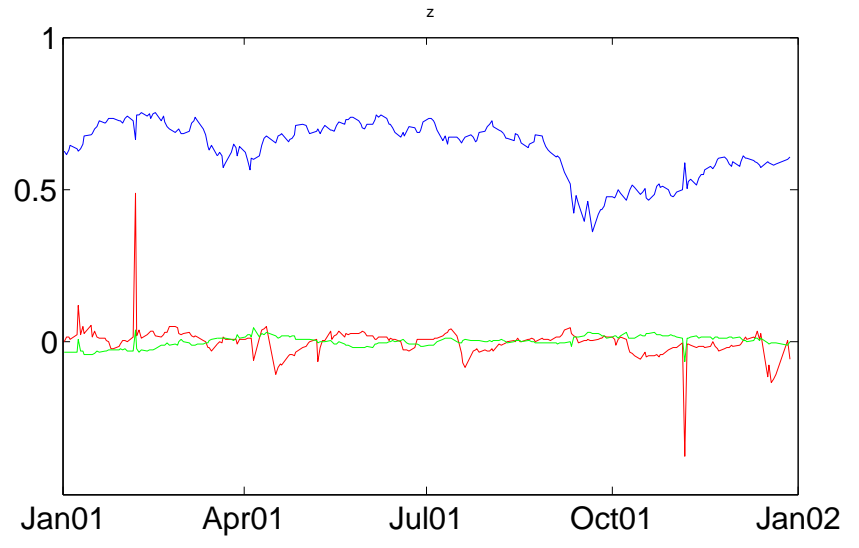

Fig. 3. Estimated $\left\{\widehat{Z}_{l t}\right\}, l=1,2,3$ (top to bottom)

In a first step we investigate the covariance structure of $\left\{\widehat{Z}_{t}\right\}$ by means of VAR analysis. Table 2 presents the parameters from the $\operatorname{VAR}(2)$ model fitted on $\left\{\widehat{Z}_{t}\right\}$. The order 2 is selected based on Akaike (AIC), Schwarz (SC) and Hannan-Quinn (HQ) criteria, see table 3. Moreover the VAR(2) model satisfies stationarity as the roots of the characteristic polynomial lie inside of the unit circle.

A natural issue is to analyse the dependences between $\left\{Z_{t}\right\}$ and the shape of the RN surfaces $\left\{\widehat{\mathcal{H}}_{t}\right\}$. In order to investigate this relation we compute the skewness $\gamma$ and excess kurtosis $\eta$ of $\widehat{q}_{t, T}\left(\cdot \mid s_{t}\right)$ across $t$ and maturities $\tau$. Here 

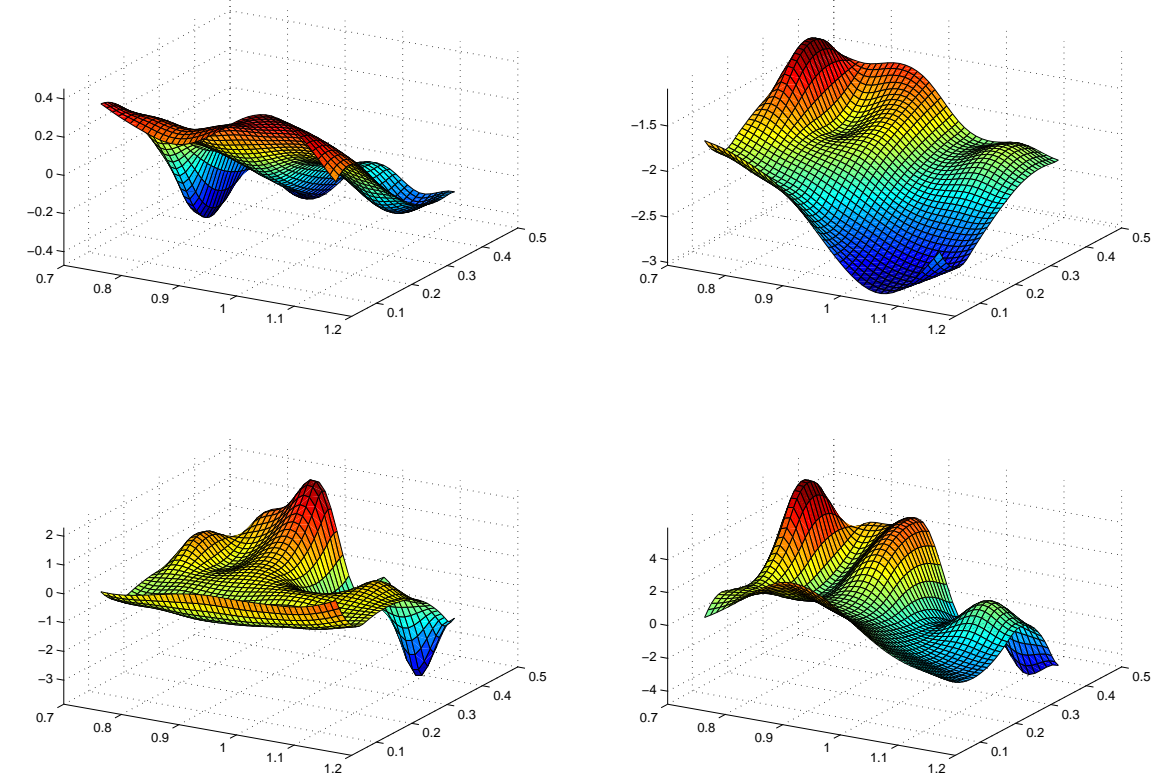

Fig. 4. Estimated basis functions $\widehat{m}_{l}, l=0, \ldots, 3$ clockwise

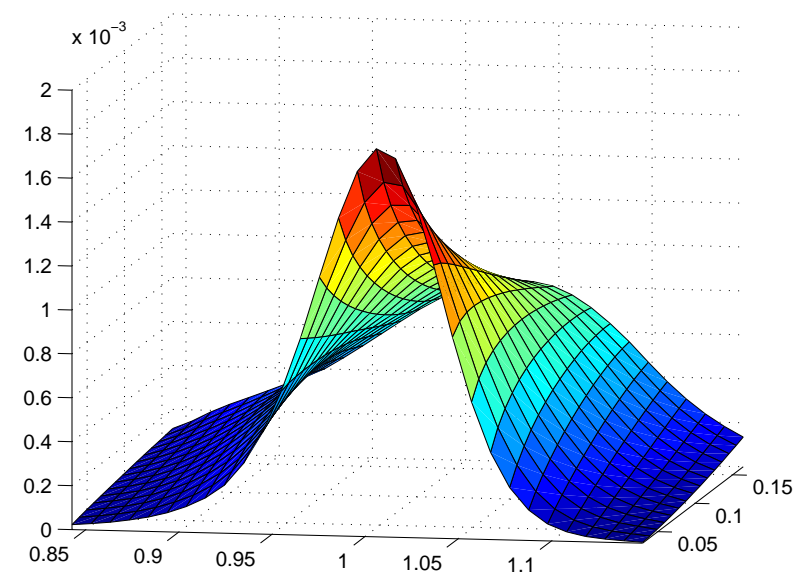

Fig. 5. Estimated RN surface, $\widehat{\mathcal{H}}_{t}$ at $t$ corresponding to day 20010710

\begin{tabular}{l|rrrrrrr}
\hline \hline & \multicolumn{7}{|c}{$\operatorname{VAR}(2)$} \\
\hline & const & $\widehat{Z}_{1, t-1}$ & $\widehat{Z}_{1, t-2}$ & $\widehat{Z}_{2, t-1}$ & $\widehat{Z}_{2, t-2}$ & $\widehat{Z}_{3, t-1}$ & $\widehat{Z}_{3, t-2}$ \\
$\widehat{Z}_{1 t}$ & 0.01 & 1.09 & -0.16 & 0.10 & -0.36 & 0.32 & -0.23 \\
$\widehat{Z}_{2 t}$ & 0.01 & -0.27 & 0.26 & 0.31 & 0.12 & -1.14 & 0.33 \\
$\widehat{Z}_{3 t}$ & 0.01 & -0.08 & 0.62 & -0.05 & -0.04 & 0.41 & 0.35 \\
\hline \hline
\end{tabular}

Table 2

Estimated parameters for the $\operatorname{VAR}(2)$ model on $\left\{\widehat{Z}_{t}\right\}$ 


\begin{tabular}{c|lll}
\hline \hline order & AIC & SC & HQ \\
\hline 1 & -11.03 & -10.99 & -11.01 \\
2 & -15.71 & $-15.54^{*}$ & -15.64 \\
3 & $-15.77^{*}$ & -15.46 & $-15.64^{*}$ \\
4 & -15.76 & -15.32 & -15.58 \\
5 & -15.72 & -15.16 & -15.45 \\
\hline \hline
\end{tabular}

Table 3

Lag selection criteria for VAR models on $\left\{\widehat{Z}_{t}\right\}$
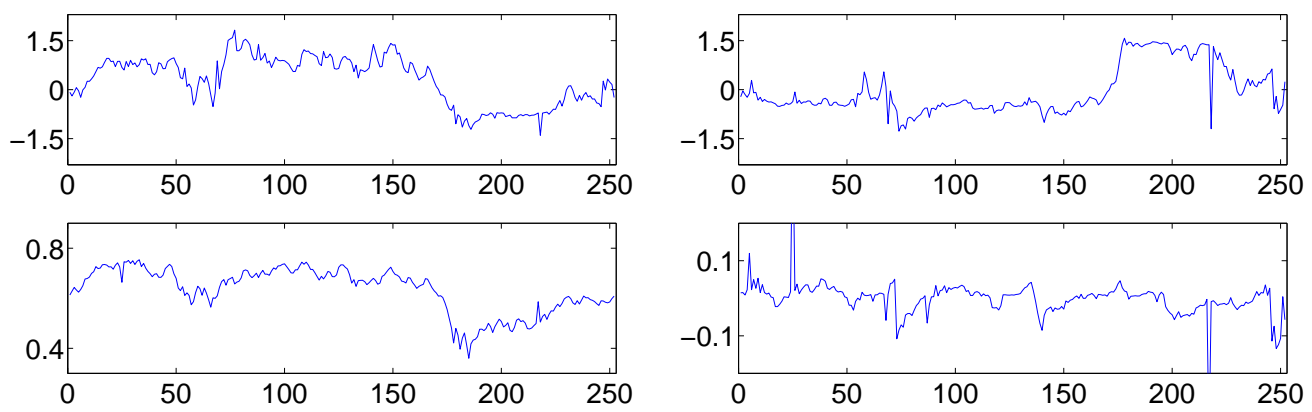

Fig. 6. Left: RN excess kurtosis $\left\{\eta_{t}\right\}, \tau=18$ (top), $\left\{\widehat{Z}_{1 t}\right\}$ (bottom) Right: RN skewness $\left\{\gamma_{t}\right\}, \tau=18$ (top), $\left\{\widehat{Z}_{2 t}\right\}$ (bottom)

$\widehat{q}_{t, T}\left(\cdot \mid s_{t}\right)=\widehat{\mathcal{H}}_{t}(\cdot, \tau)$. Figure 6 displays skewness $\left\{\gamma_{t}\right\}$ and excess kurtosis $\left\{\eta_{t}\right\}$ associated with $\widehat{q}_{t, T}$ for maturity $\tau=18$ days together with $\left\{\widehat{Z}_{1 t}\right\}$ and $\left\{\widehat{Z}_{3 t}\right\}$ and motivates the investigation of their joint autocovariance structure.

The dynamic structure of the pairs $\left\{\left(\widehat{Z}_{1 t}, \eta_{t}\right)\right\}$ and $\left\{\left(\widehat{Z}_{3 t}, \gamma_{t}\right)\right\}$ for $\tau=18$ is modelled by $\operatorname{VAR}(2)$ models. The choice of the VAR order is again based on AIC, SC, and HQ selection criteria. Portmanteau and LM tests on VAR residuals reject autocorrelations up to lag 12 and the roots of the characteristic polynomial lie inside of the unit circle.

\begin{tabular}{r|rrrrr}
\hline \hline & \multicolumn{5}{|c}{$\operatorname{VAR}(2)$} \\
\hline & const & $\widehat{Z}_{1, t-1}$ & $\widehat{Z}_{1, t-2}$ & $\eta_{t-1}$ & $\eta_{t-2}$ \\
$\widehat{Z}_{1 t}$ & 0.04 & 0.86 & 0.08 & 0.01 & 0.00 \\
$\eta_{t}$ & -0.51 & 2.63 & -1.75 & 0.67 & 0.19 \\
\hline \hline
\end{tabular}

Table 4

Estimated parameters for the $\operatorname{VAR}(2)$ model on $\left\{\left(\widehat{Z}_{1 t}, \eta_{t}\right)\right\}$

Modelling the dynamics of risk neutral densities using DSFM allows to quantify the mechanisms governing risk perceptions from agents acting in a market. Insights are obtained in two directions, concerning the autocovariance 


\begin{tabular}{r|rrrrr}
\hline \hline & \multicolumn{5}{|c}{$\operatorname{VAR}(2)$} \\
\hline & const & $\widehat{Z}_{3, t-1}$ & $\widehat{Z}_{3, t-2}$ & $\gamma_{t-1}$ & $\gamma_{t-2}$ \\
$\widehat{Z}_{3 t}$ & 0.00 & 0.20 & 0.27 & 0.01 & -0.02 \\
$\gamma_{t}$ & 0.00 & -1.69 & 0.68 & 0.81 & 0.24 \\
\hline \hline
\end{tabular}

Table 5

Estimated parameters for the $\operatorname{VAR}(2)$ model on $\left\{\left(\widehat{Z}_{3 t}, \gamma_{t}\right)\right\}$

structure of $\left\{\widehat{Z}_{t}\right\}$, i.e. the time behaviour of the RN surfaces and their crosscorrelation with the skewness and excess kurtosis from the estimated risk neutral densities, i.e. the relation between the dynamics and shape of the obtained RN surfaces. As seen in tables (4) and (5) the excess kurtosis and skewness from $\widehat{q}_{t, T}$ at maturity $\tau=18$ are determined by the corresponding lagged values of $\widehat{Z}_{t}$.

Based on the presented methodology it is possible to investigate the dynamics of the risk neutral skewness and excess kurtosis based on statistical inference on $\left\{\widehat{Z}_{t}\right\}$. A natural further step is to perform econometric analysis to investigate the cointegration between the lower dimensional time series and macroeconomic and financial indicators. This could provide deeper insights into the relation between risk assessments from investors acting in a market and the flow of economic information at which they are exposed.

\section{Acknowledgements}

Financial support from the Deutsche Forschungsgemeinschaft via SFB 649 "Economic Risk" is gratefully acknowledged.

\section{A Assumptions}

The results from Theorems (2.1) and (2.2), see Borak et al. (2008) rely on the following assumptions:

(A1) The variables $X_{11}, \ldots, X_{J T}, \varepsilon_{11}, \ldots, \varepsilon_{J T}$ and $Z_{1}, \ldots, Z_{T}$ are independent. The process $Z_{t}$ is allowed to be nonrandom.

(A2) For $t=1, \ldots, T$ the variables $X_{1 t}, \ldots, X_{J t}$ are identically distributed, have support $[0,1]^{d}$ and a density $f_{t}$ that is bounded from below and above on $[0,1]^{d}$, uniformly over $t=1, \ldots, T$. 
(A3) We assume that $E\left[\varepsilon_{j t}\right]=0$ for $t=1, \ldots, T$ and $j=1, \ldots, J$ and

$$
\sup _{t=1, \ldots, T, j=1, \ldots, J} E \exp \left[c \varepsilon_{j t}^{2}\right]<\infty
$$

for $c>0$ small enough.

(A4) The functions $\psi_{k}$ may depend on the increasing indices $T$ and $J$ and are normed so that $\int_{[0,1]^{d}} \psi_{k}^{2}(x) d x=1$ for $k=1, \ldots, K$. Furthermore it holds that $\sup _{x \in[0,1]}\|\psi(x)\|=\mathcal{O}\left(K^{1 / 2}\right)$

(A5) The components $m_{0}, \ldots, m_{L}$ can be approximated by $\psi_{1}, \ldots, \psi_{K}$, i.e.

$$
\delta_{K}=\sup _{x \in[0,1]^{d}} \inf _{\Gamma \in \mathcal{G}}|m(x)-\Gamma \psi(x)| \rightarrow 0
$$

for $l=0, \ldots, L$ and $K \rightarrow \infty$. We denote by $\Gamma^{*}$ the matrix that fulfills

$$
\sup _{x \in[0,1]^{d}}|m(x)-\Gamma \psi(x)| \leq 2 \delta_{K}
$$

(A6) There exist constants $0<C_{L}<C_{U}<\infty$ such that all eigenvalues of the random matrix $T^{-1} \sum_{t=1}^{T} Z_{t} Z_{t}^{\top}$ lie in the interval $\left[C_{L}, C_{U}\right]$ with probability tending to one.

(A7) The minimization (2.2) runs over all values of $(\Gamma, z)$ with

$$
\sup _{x \in[0,1]} \max _{1 \leq t \leq T}\left\|Z_{t}^{\top} \Gamma \psi(x)\right\| \leq M_{T}
$$

where $M_{T}$ fulfills $\max _{1 \leq t \leq T}\left\|Z_{t}\right\| \leq M_{T} / C_{m}$ (with probability tending to one) for a constant $C_{m}>\sup _{x \in[0,1]}\|m(x)\|$.

(A8) It holds that $\rho^{2}=(K+T) M_{T}^{2} \log \left(J T M_{T}\right) /(J T) \rightarrow 0$. The dimension $L$ is fixed.

(A9) $Z_{t}$ is a martingale difference with $E\left[Z_{t} \mid Z_{1}, \ldots, Z_{t_{1}}\right]=0$ and for some $C>0$ $E\left[\|\left. Z_{t}\right|^{2} \mid Z_{1}, \ldots, Z_{t_{1}}\right]<C$ (a.s). The matrix $E\left[Z_{t} Z_{t}^{\top}\right]$ has full rank. The process $Z_{t}$ is independent of $X_{11}, \ldots, X_{T J}$ and $\varepsilon_{11}, \ldots, \varepsilon_{T J}$.

(A10) The functions $m_{0}, \ldots, m_{L}$ are linearly independent. In particular, no function is equal to 0 .

(A11) It holds that $\left(K^{1 / 2} M_{T}+T^{1 / 4}\right)\left(\rho+\delta_{K}\right)=\mathcal{O}(1)$.

\section{References}

Ait-Sahalia, Y., and Lo, A. (1998), "Nonparametric estimation of state-price densities implicit in financial asset prices," Journal of Finance, 53, 499-547.

Benko, M., Kneip, A., and Härdle, W. (2008), "Common Functional Principal Components," Annals of Statistics, forthcoming.

Besse, P., Cardot, H., and Ferraty, F. (1997), "Some Tools for Functional Data Analysis," Computational Statistics and Data Analysis, 24, 255-270. 
Borak, S., Härdle, W., Mammen, E., and Park, B. (2008), "Time series modelling with semiparametric factor dynamics," Journal of the American Statistical Association, submitted.

Breeden, D., and Litzenberger, R. (1978), "Prices of state-contingent claims implicit in options prices," Journal of Business, 51, 621-651.

Brüggemann, R., Härdle, W., Mungo, J., and Trenkler, C. (2008), "VAR Modeling for Dynamic Loadings Driving Volatility Strings," Journal of Financial Econometrics, Advance Access.

Cont, R., and da Fonseca, J. (2002), "The Dynamics of Implied Volatility Surfaces," Quantitative Finance, 2, 45-60.

Dähne, S., Härdle, W., and Hautsch, H. (2008), "Quantification of Liquidity Costs: Forecasting the Ordebook," Discussion Paper Series SFB 649, Humboldt-Universität zu Berlin.

Dauxois, J., Pousse, A., and Romain, Y. (1982), "Asymptotic Theory for the Principal Component Analysis of a Vector Random Function: Some Applications to Statisticsl Inference," Journal of Multivariate Analysis, 12, $136-154$

Fengler, M., Härdle, W., and Mammen, E. (2007), "A semiparametric factor model for implied volatility surface dynamics," Journal of Financial Econometrics, 5, 189-218.

Ferraty, F., and Vieu, P. (2006), Nonparametric Functional Data Analysis, Springer, New York.

Gasser, T., and Kneip, A. (1995), "Searching for Structure in Curve Samples," Journal of the American Statistical Association, 90(432), 1179-1188.

Hernández-Hernández, D., and Schied, A. (2007), "A control approach to robust maximization with logarithmic utility and time-consistent penalties," Stochastic Processes and Their Applications, 117(8), 980-1000.

Kneip, A. (1994), "Nonparametric Estimation of Common Regressors for Similar Curve Data," Annals of Statistics, 22(3), 1386-1427.

Kneip, A., and Gasser, T. (1992), "Statistical Tools to Analyse Data Representing a Sample of Curves," Annals of Statistics, 20(3), 1266-1305.

Ramsay, J. O., and Dalzell, C. T. (1991), "Some Tools for Functional Data Analysis," Journal of the Royal Statistical Society B, 53(3), 539-572.

Ramsay, J. O., and Silverman, B. W. (2005), Functional Data Analysis, Springer, New York.

Rao, C. (1958), "Some Statistical Methods for Comparison of Growth Curves," Biometrics, 14, 434-471.

Rice, J., and Silverman, B. W. (1991), "Estimating the Mean and Covariance Structure Nonparametrically when the Data are Curves," Journal of Royal Statistics Society B, 53, 233-243. 


\section{SFB 649 Discussion Paper Series 2008}

For a complete list of Discussion Papers published by the SFB 649, please visit http://sfb649. wiwi. hu-berlin.de.

001 "Testing Monotonicity of Pricing Kernels" by Yuri Golubev, Wolfgang Härdle and Roman Timonfeev, J anuary 2008.

002 "Adaptive pointwise estimation in time-inhomogeneous time-series models" by Pavel Cizek, Wolfgang Härdle and Vladimir Spokoiny, January 2008.

003 "The Bayesian Additive Classification Tree Applied to Credit Risk Modelling" by Junni L. Zhang and Wolfgang Härdle, January 2008.

004 "Independent Component Analysis Via Copula Techniques" by Ray-Bing Chen, Meihui Guo, Wolfgang Härdle and Shih-Feng Huang, January 2008.

005 "The Default Risk of Firms Examined with Smooth Support Vector Machines" by Wolfgang Härdle, Yuh-Jye Lee, Dorothea Schäfer and Yi-Ren Yeh, January 2008.

006 "Value-at-Risk and Expected Shortfall when there is long range dependence" by Wolfgang Härdle and J ulius Mungo, Januray 2008.

007 "A Consistent Nonparametric Test for Causality in Quantile" by Kiho Jeong and Wolfgang Härdle, January 2008.

008 "Do Legal Standards Affect Ethical Concerns of Consumers?" by Dirk Engelmann and Dorothea Kübler, January 2008.

009 "Recursive Portfolio Selection with Decision Trees" by Anton Andriyashin, Wolfgang Härdle and Roman Timofeev, January 2008.

010 "Do Public Banks have a Competitive Advantage?" by Astrid Matthey, January 2008.

011 "Don't aim too high: the potential costs of high aspirations" by Astrid Matthey and Nadja Dwenger, J anuary 2008.

012 "Visualizing exploratory factor analysis models" by Sigbert Klinke and Cornelia Wagner, January 2008.

013 "House Prices and Replacement Cost: A Micro-Level Analysis" by Rainer Schulz and Axel Werwatz, January 2008.

014 "Support Vector Regression Based GARCH Model with Application to Forecasting Volatility of Financial Returns" by Shiyi Chen, Kiho Jeong and Wolfgang Härdle, January 2008.

015 "Structural Constant Conditional Correlation" by Enzo Weber, January 2008.

016 "Estimating Investment Equations in Imperfect Capital Markets" by Silke Hüttel, Oliver Mußhoff, Martin Odening and Nataliya Zinych, January 2008.

017 "Adaptive Forecasting of the EURIBOR Swap Term Structure" by Oliver Blaskowitz and Helmut Herwatz, January 2008.

018 "Solving, Estimating and Selecting Nonlinear Dynamic Models without the Curse of Dimensionality" by Viktor Winschel and Markus Krätzig, February 2008.

019 "The Accuracy of Long-term Real Estate Valuations" by Rainer Schulz, Markus Staiber, Martin Wersing and Axel Werwatz, February 2008.

020 "The Impact of International Outsourcing on Labour Market Dynamics in Germany" by Ronald Bachmann and Sebastian Braun, February 2008.

021 "Preferences for Collective versus Individualised Wage Setting" by Tito Boeri and Michael C. Burda, February 2008.

\section{SFB 649, Spandauer Straße 1, D-10178 Berlin} http:/ / sfb649.wiwi.hu-berlin.de

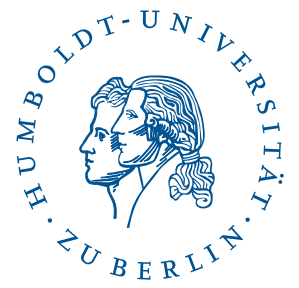


022 "Lumpy Labor Adjustment as a Propagation Mechanism of Business Cycles" by Fang Yao, February 2008.

023 "Family Management, Family Ownership and Downsizing: Evidence from S\&P 500 Firms" by J örn Hendrich Block, February 2008.

024 "Skill Specific Unemployment with Imperfect Substitution of Skills" by Runli Xie, March 2008.

025 "Price Adjustment to News with Uncertain Precision" by Nikolaus Hautsch, Dieter Hess and Christoph Müller, March 2008.

026 "Information and Beliefs in a Repeated Normal-form Game" by Dietmar Fehr, Dorothea Kübler and David Danz, March 2008.

027 "The Stochastic Fluctuation of the Quantile Regression Curve" by Wolfgang Härdle and Song Song, March 2008.

028 "Are stewardship and valuation usefulness compatible or alternative objectives of financial accounting?" by J oachim Gassen, March 2008.

029 "Genetic Codes of Mergers, Post Merger Technology Evolution and Why Mergers Fail" by Alexander Cuntz, April 2008.

030 "Using R, LaTeX and Wiki for an Arabic e-learning platform" by Taleb Ahmad, Wolfgang Härdle, Sigbert Klinke and Shafeeqah Al Awadhi, April 2008.

031 "Beyond the business cycle - factors driving aggregate mortality rates" by Katja Hanewald, April 2008.

032 "Against All Odds? National Sentiment and Wagering on European Football" by Sebastian Braun and Michael Kvasnicka, April 2008.

033 "Are CEOs in Family Firms Paid Like Bureaucrats? Evidence from Bayesian and Frequentist Analyses" by Jörn Hendrich Block, April 2008.

034 "JBendge: An Object-Oriented System for Solving, Estimating and Selecting Nonlinear Dynamic Models" by Viktor Winschel and Markus Krätzig, April 2008.

035 "Stock Picking via Nonsymmetrically Pruned Binary Decision Trees" by Anton Andriyashin, May 2008.

036 "Expected Inflation, Expected Stock Returns, and Money Illusion: What can we learn from Survey Expectations?" by Maik Schmeling and Andreas Schrimpf, May 2008.

037 "The Impact of Individual Investment Behavior for Retirement Welfare: Evidence from the United States and Germany" by Thomas Post, Helmut Gründl, Joan T. Schmit and Anja Zimmer, May 2008.

038 "Dynamic Semiparametric Factor Models in Risk Neutral Density Estimation" by Enzo Giacomini, Wolfgang Härdle and Volker Krätschmer, May 2008.

\section{SFB 649, Spandauer Straße 1, D-10178 Berlin} http:/ / sfb649.wiwi.hu-berlin.de

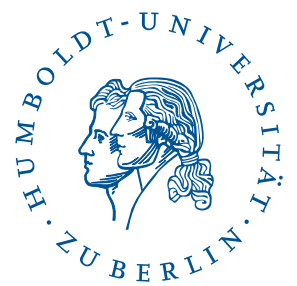

